\title{
CaNaPy: SatComm LGS-AO experimental platform with laser uplink pre-compensation
}

D. Bonaccini Calia, M. Centrone, E. Pinna, D. Alaluf, N. Martinez, et al.

D. Bonaccini Calia, M. Centrone, E. Pinna, D. Alaluf, N. Martinez, J. Osborn, W. Hackenberg, M. Townson, M. Faccini, A. Di Paola, L. F. Rodriguez Ramos, R. Speziali, M. Reyes, P. Janout, D. Jenkins, D. P. Wei, T. Fischer, J. Perdigues, W. R. L. Clements, "CaNaPy: SatComm LGS-AO experimental platform with laser uplink pre-compensation," Proc. SPIE 11852, International Conference on Space Optics - ICSO 2020, 118521A (11 June 2021); doi: $10.1117 / 12.2599233$ SPIE. Event: International Conference on Space Optics - ICSO 2021, 2021, Online 


\section{International Conference on Space Optics-ICSO 2020}

Virtual Conference

30 March-2 April 2021

Edited by Bruno Cugny, Zoran Sodnik, and Nikos Karafolas
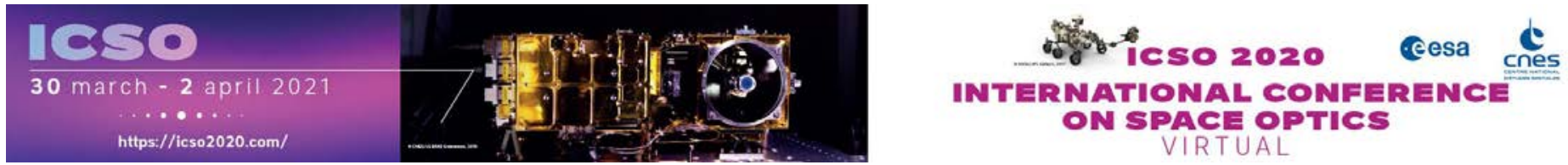

\section{CaNaPy: SatComm LGS-AO experimental platform with laser uplink pre-compensation}

\section{Cesa isoporecestings denes}




\title{
CaNaPy: SatComm LGS-AO experimental platform with laser uplink pre-compensation
}

\author{
D. Bonaccini Calia ${ }^{\mathrm{a}}$, M. Centrone ${ }^{\mathrm{b}}$, E.Pinna ${ }^{\mathrm{g}}$, D.Alaluf ${ }^{\mathrm{e}}$, N.Martinez $^{\mathrm{h}}$, J.Osborn $^{\mathrm{c}}$, W.Hackenberg ${ }^{\mathrm{a}}$, \\ M.Townson ${ }^{c}$, M.Faccini ${ }^{b}$, A.Di Paola ${ }^{\text {b }}$, L.F.Rodriguez Ramos ${ }^{\text {d }}$, R.Speziali ${ }^{\text {b }}$, M.Reyes ${ }^{\text {d }}$, P.Janout ${ }^{\mathrm{a}}$, \\ D.Jenkins $^{\text {a }}$, D.P.Wei ${ }^{\mathrm{f}}$, T.Fischer ${ }^{\mathrm{i}}$, J. Perdigues ${ }^{\mathrm{e}}$ W.R.L. Clements ${ }^{\mathrm{f}}$ \\ ${ }^{a}$ European Southern Observatory, Karl-Schwarzschildstr. 2, 85748 Garching, Germany; \\ ${ }^{\mathrm{b}}$ INAF Osservatorio Astronomico di Roma, Via Frascati 33, 00078 Monte Porzio Catone (Roma), Italy; \\ ${ }^{\mathrm{c}}$ Centre for Advanced Instrumentation, Physics Dept., University of Durham, South Road, Durham DH1 3LE, UK; \\ 'Istituto de Astrofisica Canarias, Vía Láctea, s/n, 38205 San Cristóbal de La Laguna, Santa Cruz de Tenerife, España; \\ ${ }^{\text {e }}$ ESA/ESTEC, Keplerlaan 1, 2200 AG Noordwijk ZH, Netherlands. \\ ${ }_{\mathrm{f}}^{\mathrm{f}}$ MPB Communications, 147 Hymus Blvd, Pointe-Claire, Quebec H9R 1E9, Canada \\ ${ }^{g}$ INAF Osservatorio Astronomico di Arcetri, L.go E.Fermi 5, 50125 Firenze, Italy \\ ${ }^{\text {h }}$ Australian National University Stromlo Observatory, Cotter Road, Weston Creek 2611 ACT - Australia \\ ${ }^{\mathrm{i}}$ HighFinesse GmbH, Wöhrdstr. 4, 72072 Tübingen, Germany
}

\begin{abstract}
We report on the visible LGS-AO experimental facility which we are building to be installed at the 1m ESA Optical Ground Station at Observatorio del Teide, Tenerife, Canary Islands. We focus on the system aspects related to optics.

The instrument will be a novel facility to perform strategic LGS-AO technology R\&D in future years, demonstrating a $50+$ W CW 589nm laser, uplink laser beam pre-compensation on sodium LGS in pulsed laser operation.

The rationale for the LGS-AO facility is to have a synergy between astronomical and space awareness adaptive optics, to perform experiments related to LGS-AO technologies for astrophysics and optical communication with satellites (Optical Feeder Links), including daytime LGS-AO, for future OGS systems.
\end{abstract}

Keywords: Optical Communications, Laser Guide Stars, Adaptive Optics

\section{INTRODUCTION}

Adaptive Optics with laser guide star (LGS-AO) has been in routine use at large astronomical telescope facilities since more than two decades ${ }^{1,2}$. However, it is only relatively recently that reliable and stable second generation LGS-AO systems have been installed and used routinely at major astronomical observatories, usually located at remote, high altitude sites, without requiring major efforts to operate them. This has been thanks to developments in the controls and in the LGS-AO subsystems. A great impact on the reliability and operability of the LGS-AO facilities has been given by the development of turn-key 589nm, solid state, narrow band lasers ${ }^{3}$ based on 36W CW Raman Fiber Amplifiers ${ }^{4}$, with powers of $22 \mathrm{~W} \mathrm{CW}$ or higher, incorporating features allowing efficient sodium optical pumping, including repumper photons and accurate wavelength stabilization within $2 \mathrm{MHz}$ rms. Recently MPBC has been able, in a collaborative effort with ESO, to produce 95W CW RFA, which leads to an LGS laser with more than 50W CW at 589nm.

The choice of lasers emitting at D2a sodium wavelengths (589.16nm in vacuum), allows to excite resonant scattering in the mesospheric atomic sodium layer, a $20 \mathrm{~km}$ thick region which is centered at around $90 \mathrm{~km}$ a.s.l., well above the atmospheric turbulence layers. The cone effect ${ }^{5}$, or focal anisoplanatism, is very small with high altitude LGS, such as sodium LGS, compared to Rayleigh scattering LGS, which are positioned at 10-15 km above the site. 


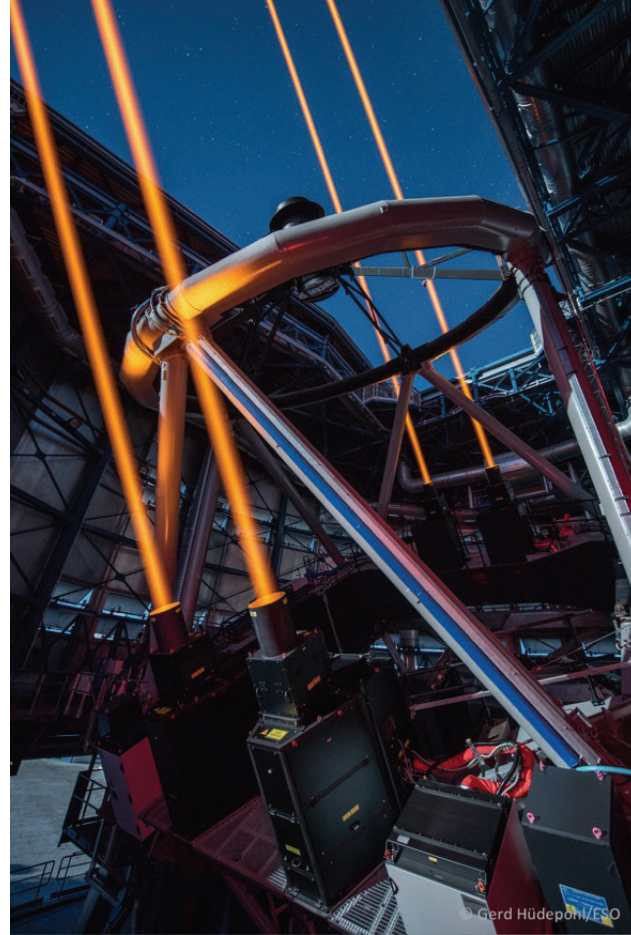

Figure 1: the 4LGS facility in operation since 2016 at the ESO Paranal Observatory (Chile)

Several years of experience have been gained by some of the authors institutes, producing operational LGS-AO facilities for high spatial resolution astrophysics ${ }^{6}$. This has resulted in turn-key LGS-AO facilities, run by telescope operators, in routine operation at the ESO VLT Yepun 8m telescope in Cerro Paranal (Chile).

In a collaborative ESO-ESA agreement, the synergy on R\&D for LGSAO between the two institutes has been identified as of high priority. Some of the novel LGS-AO systems requirements needed for optical feeder links, are co-aligned with the technology research goals for astronomy, to extend the LGS-AO sky coverage to $100 \%$, perform full uplink pre-compensation as well as daytime, or high turbulence conditions, operation (for solar and for thermal band astrophysics ${ }^{7,8}$ ).

The LGS-AO application of extreme interest for this community is for optical feeder links (OFL), solving the point ahead problem. The resulting full uplink pre-compensation of the OFL brings a stronger communication signal at the satellite when the phase is well corrected, as shown in our simulation section. This application of LGS-AO, from optical ground stations to satellites, introduces extra requirements which are not usual for the systems built so far in the astronomical community. These are:

24/7 operation. The daytime LGS-AO operation requires efficient sky background suppression, which we have demonstrated possible using a $0.1 \AA$ filter. Especially in the late morning and afternoon hours, the turbulence conditions require more spatial and spectral resolution in the adaptive optics correction, which translates into faster $\mathrm{AO}$ closed loop operation (1-2 kHz for GEO, 3-5kHz for LEO), more actuators in the deformable mirrors, higher LGS brightness, hence higher laser power. Extreme turbulent conditions have implications on scintillation and optical phase behavior. Thus, the operation of LGS-AO in extreme turbulent conditions requires dedicated R\&D to be addressed experimentally and is a challenge at system level, as of today's technologies.

\begin{tabular}{|c|c|c|c|c|c|c|c|}
\hline \multicolumn{4}{|c|}{ seeing (500nm) at Zenith: $0.7^{\prime \prime}$} & \multicolumn{4}{|c|}{ seeing (500nm) at Zenith: $2.0^{\prime \prime}$} \\
\hline Parameter & $589 \mathrm{~nm}$ & $1064 \mathrm{~nm}$ & $1550 \mathrm{~nm}$ & Parameter & $589 \mathrm{~nm}$ & $1064 \mathrm{~nm}$ & $1550 \mathrm{~nm}$ \\
\hline Elevation $\left[{ }^{\circ}\right]$ & 30 & 30 & 30 & Elevation $\left[{ }^{\circ}\right]$ & 30 & 30 & 30 \\
\hline Seeing ["] & 1.03 & 0.91 & 0.85 & Seeing ["] & 2.94 & 2.61 & 2.42 \\
\hline$r_{0}(\lambda)[\mathrm{cm}]$ & 12 & 24 & 38 & $r_{0}(\lambda)[\mathrm{cm}]$ & 4 & 8 & 13 \\
\hline$\tau_{\circ}(\lambda)[\mathrm{ms}]$ & 2.8 & 5.6 & 8.9 & $\tau_{\mathrm{o}}(\lambda)[\mathrm{ms}]$ & 0.9 & 1.8 & 2.8 \\
\hline$\nu_{\text {Loop }}(\mathrm{kHz})$ & 1.2 & 0.6 & 0.37 & $\nu_{\text {Loop }}(\mathrm{kHz})$ & 3.3 & 1.7 & 1.0 \\
\hline$\theta_{\text {isok }}["]$ & 2.9 & 4.8 & 7.2 & $\theta_{\text {isok }}[$ ["] & 1.2 & 2.0 & 3.12 \\
\hline$d_{o}[m]$ & 3.51 & 7.13 & 11.2 & $d_{o}[m]$ & 1.23 & 2.50 & 3.92 \\
\hline$\theta_{\text {Cone }}["]$ & & 0.86 & & $\theta_{\text {Cone }}["]$ & & 3.183 & \\
\hline
\end{tabular}

Figure 2: main LGS-AO parameters, for the Tenerife model atmosphere, for seeing conditions, at Zenith and at 500nm, of 0.7 " and 2.0", respectively, and for pointing elevation of $30 \mathrm{deg}$. The values are given for three representative wavelengths. The cone effect of the sodium LGS is represented by $\mathrm{d}_{\mathrm{o}}$ and by $\theta_{\text {cone }}$. 
The parameter $\theta_{\text {Cone }}$ in Figure 2 represents the point ahead angle (PAA, in arcsec), above which the cone effect has smaller impact that the isoplanatic effect. In other words, at which PAA the use of the satellite downlink reference for AO is worse than using the LGS. It does not depend on wavelength, as both scale the same way. This analytical result shows that e.g., for LEO (and in some cases for GEO) at 30 degrees elevation angle, the use of LGS instead of the satellite itself as reference for the AO system is always advantageous. This is reflected also in the numerical simulations given in the sections below.

Scintillation induced by the turbulence creates a variability of the optical feeder signal at the target satellite, which impacts the error rates, and it is not at all easy to correct for. It can be however mitigated by the averaging effect induced on a larger aperture, if the uplink optical feeder laser beam is composed by temporally incoherent patches across the pupil.

Tip-tilt may be detected from the satellite, if the point ahead angle is smaller than the isokinetic angle. In case it isn't, the uplink tilt has to be derived from the LGS itself. This is more mandatory e.g. for LEO applications. Although several methods have been proposed ${ }^{9,10,11}$, none have been really tested experimentally in depth. They have to be addressed, especially for non-geostationary satellites. This is one of the goals of CaNaPy in the ESO-ESA collaboration. The capability to detect the atmospheric tip-tilt from the LGS would be a breakthrough also for astrophysics application, in which the LGS-AO has to resorts to available and sufficiently bright natural guide stars, which limits the sky coverage of LGS-AO in astronomy.

To this extent, feasibility experiments have been done in the past two years by some of the authors, using the $20 \mathrm{~W}$ $589 \mathrm{~nm}$ CW laser of the ESO WLGSU ${ }^{12}$ unit, the Isaac Newton Telescope and the Swedish Solar Tower at the Observatorio del Roque de los Muchachos, to explore one of the possible methods for uplink tip-tilt sensing via the LGS, as well as the photometry of the daytime LGS $^{13}$ (see poster 359 of this conference), in collaboration with DLR and the Swedish Solar Tower teams, respectively.

\section{CANAPY MAIN REQUIREMENTS}

The CaNaPy is designed with the following system requirements:

1. The adaptive optics loop is optimized for operation with Sodium LGS, including adaptive modal gain control.

2. Use of a $50 \mathrm{~W}$ (goal $70 \mathrm{~W}$ ) 589nm LGS laser in long pulsed mode. The laser is based on the Raman Fiber Amplifier technology.

3. A Pyramid WFS (Py-WFS) equipped with an OCAM2S camera from First Light is used with the LGS, taking advantage of the uplink correction.

4. A scoring camera is present in the Py-WFS, which can be used to optimize the loop gain or to detect long exposures of the LGS.

5. A Shack-Hartmann wavefront sensor operating with natural guide stars (NGS) is used in engineering mode to fine tune or verify, the uplink LGS operation independently of the Py-WFS. It can also be used with the LGS.

6. There is provision for IR Tx laser injection and for the IR Py-WFS to be used with the satellite source as AO reference.

7. Full pre-compensation of the uplink beam. The IR OFL laser runs superimposed with the LGS laser (small offset to compensate the delay of LGS from ground to mesosphere)

8. Monostatic propagation of the 589nm LGS laser on the OGS $1 \mathrm{~m}$ diameter pupil. Option for the IR OFL laser to use the full OGS pupil or a $30 \mathrm{~cm}$ off-axis pupil.

9. Use of double axicons to create Bessel-1 ring intensity distribution, to avoid losing uplink laser light due to the OGS central obstruction (obstruction ratio $\varepsilon=0.284$ );

10. System optimized for $r_{0}(500) \geq 10 \mathrm{~cm}$ at OGS, for 589, 1064, 1550nm. Upgrade provisions.

11. Up to $2 \mathrm{kHz}$ AO loop operation. Upgrade provisions for faster loop rates.

12. Night-time and Daytime LGS-AO (removing sky background, $0.1 \AA$ filter, see Poster 359 at this conference) 
13. Cn2 vertical distribution measurement, 24/7, to support the observations and the control

14. Laser Pointing Camera (LPC) from Astrel Instruments, capable to see the $589 \mathrm{~nm}$ and the $1064 \mathrm{~nm}$ laser beams, including the LGS. The smart camera uses the Sky as coordinate reference to give pointing offsets and LGS parameters. It is attached to a side telescope of the OGS.

\section{SYSTEM BLOCK DIAGRAM}

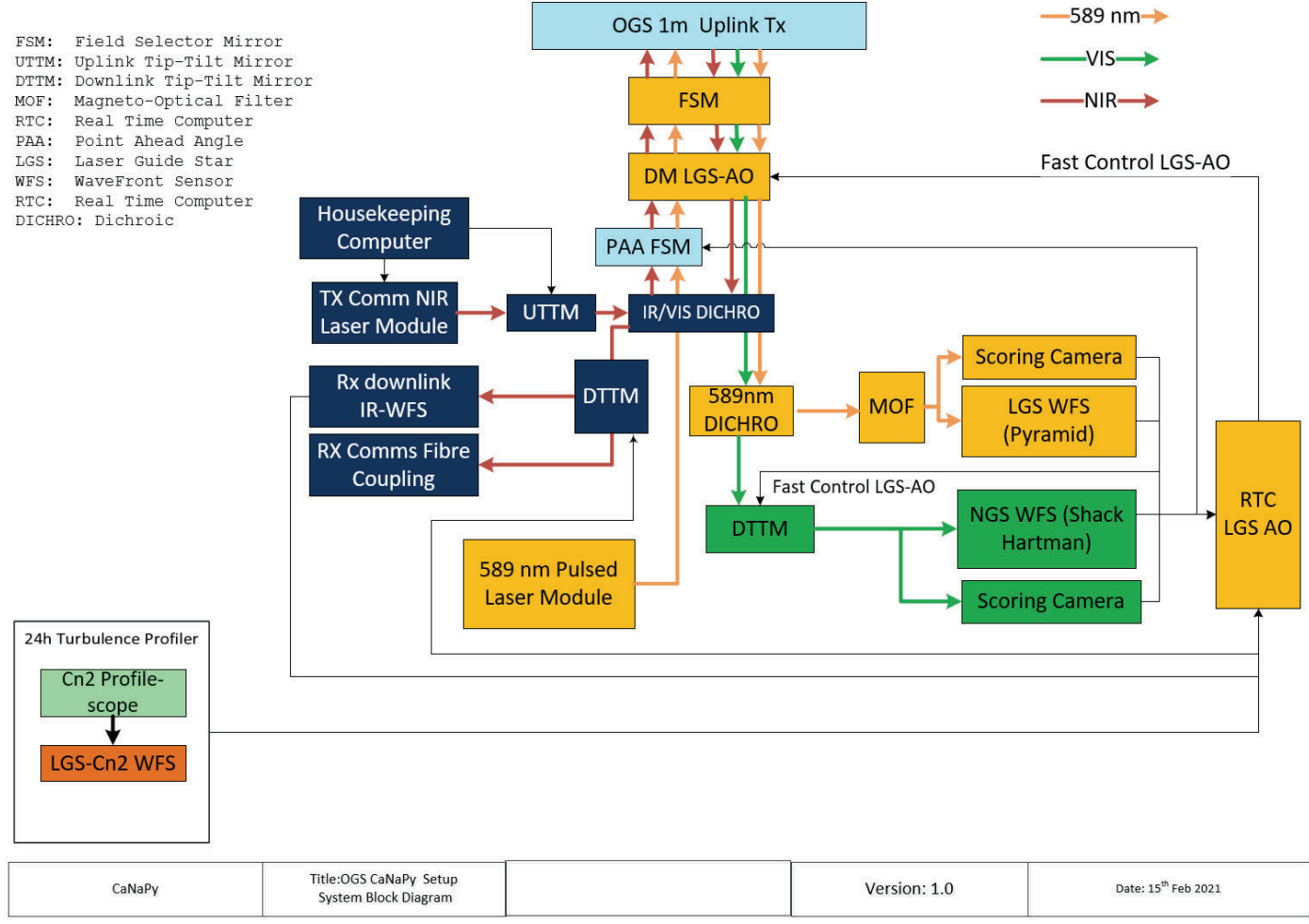

Figure 3: CaNaPy System functional block diagram. In dark blue the infrared OFL components.

To be noted in the Figure 3 functional block diagram of the CaNaPy System:

- The design enables testing OFL with full modal pre-compensation.

- There is a Shack-Hartmann WFS to be used for NGS, and a Pyramid WFS for the LGS.

- There is provision for an IR Py-WFS to be used either

$\bigcirc$ as tip-tilt (truth) sensor from the satellite, or

- standalone to close the loop with the satellite downlink signal as reference - no LGS operation. This will be used as benchmark, to be compared with the LGS-AO operated OFL.

- The use of Scoring cameras in both LGS Py-WFS and SH-WFS for the visible NGS.

- The Sodium Magneto-Optical Filter in front of the Py-WFS.

- The Real Time Computer can use any of three wavefront sensors (IR Py-WFS, 589nm Py-WFS, NGS SH-WFS) to close the AO loop, and is capable to operate in different configurations, with precompensation of beam jitter alone, or full precompensation.

- The LGS made in monostatic propagation does not need to correct for LGS jitter at the Py-WFS. 


\section{SUB-SYSTEMS}

We explore in this section some aspects of the CaNaPy subsystems which are more related with optics.

\subsection{9nm Laser}

The CaNaPy 589nm laser uses the same Raman Fiber Amplifier (RFA) technology as developed and patented at ESO in the years $2005-2009^{14}$, engineered and optimized by the Toptica-MPBC consortium in their 22W CW, "Guide Star Laser" product and deployed at a number of large observatories worldwide. The RFA scheme is shown in Figure 4 on the left.

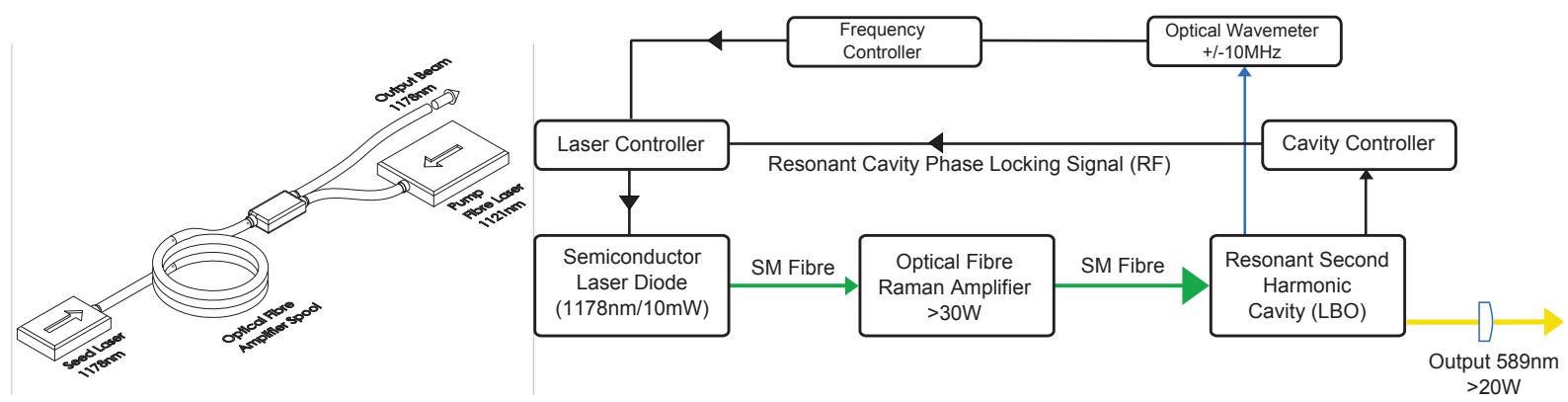

Figure 4. Left: Scheme of the $1178 \mathrm{~nm}$ source of the RFA. With respect to the commercial units at $36 \mathrm{~W}$ CW, the RFA has been enhanced in a collaborative agreement with $\mathrm{MPBC}^{\mathrm{i}}$ to produce $100 \mathrm{~W} \mathrm{CW}$. The $589 \mathrm{~nm}$ photons are obtained via resonant second harmonic generation. Right: block diagram of the CaNaPy laser.

As can be seen in Figure 4, most of the wavelength conversions are done in the fibers up to the RFA output at 1178nm, with the last frequency doubling done in an external doubly resonant Second Harmonic Generation unit from Toptica Projects Gmbh.

Within a collaborative agreement for RFA development between ESO and MPBC, the company has delivered to ESO laser laboratory a remarkable $100 \mathrm{~W}$ CW $1178 \mathrm{~nm}$ RFA, to be used in the CaNaPy $589 \mathrm{~nm}$ laser.

Optical conversion efficiencies higher than $80 \%$ have been demonstrated at our labs up to $50 \mathrm{~W} \mathrm{CW}$ at $589 \mathrm{~nm}$, as shown in Figure 5, and are routine in the 22W GSL commercial units, using the Toptica SHG doubly resonant laser cavity.

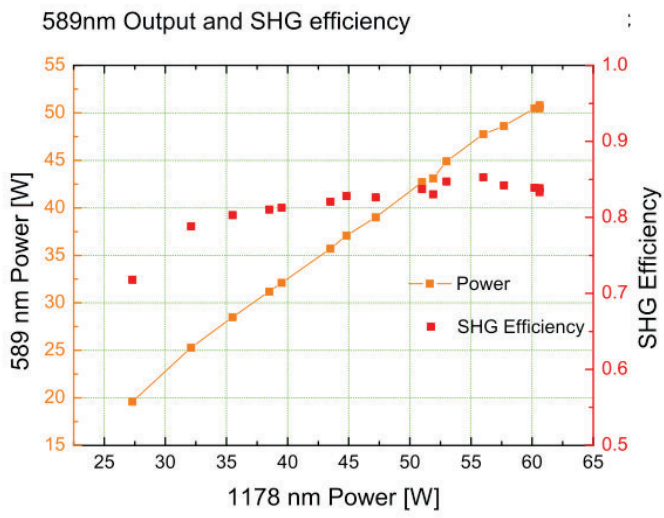

Figure 5: conversion efficiencies (red squares) higher than $80 \%$ have been demonstrated at ESO laser laboratory, for $589 \mathrm{~nm}$ powers of $50 \mathrm{~W}$ and higher (orange squares).

\footnotetext{
${ }^{\mathrm{i}}$ https://www.mpbcommunications.com/
} 
The scalability of the 589nm laser based on RFA technologies has been demonstrated, with power levels of $70 \mathrm{~W}$ CW at $589 \mathrm{~nm}$ as a goal to be demonstrated. A TRL6 589nm laser with more than 50W CW power at 589nm is being assembled at ESO laser labs at the time of this writing. The laser has the correct properties for optimal LGS brightness, based on our past experience.

The laser emission for the LGS maximum efficiency needs to have a repumper line, i.e. a fraction of the emitted photons shifted by $1.73 \mathrm{GHz}$ towards the blue of the $\mathrm{D}_{2 \mathrm{a}}$ sodium line; it needs to be frequency stabilized within $\pm 10 \mathrm{MHz}$ and circularly polarized. Frequency chirping, developed in a collaborative effort with Toptica Projects Gmbh, is the option used to avoid spectral hole burning and saturation of the mesospheric sodium atoms at higher power densities. Moreover, pulsed operation at $589 \mathrm{~nm}$ to allow monostatic uplink beam pre-compensation is introduced as an option.

The 589nm laser frequency is absolutely referenced and controlled using a new, precision optical wavemeter from HighFinesse $^{\mathrm{ii}}$, with $2 \mathrm{MHz}$ rms error and multiple wavelength stabilization channels, acting on the $1178 \mathrm{~nm}$ seed laser of the RFA. The unit can monitor and control at the same time also up to 6 more laser wavelengths (e.g. the OFL IR emission lasers), operating in CW or pulsed mode.

\subsection{Pyramid Wavefront Sensor}

The use of a Pyramid wavefront sensor ${ }^{15}$ for both, the LGS and the satellite downlink signal is optimal with the full uplink pre-compensation of the LGS. The Pyramid wavefront sensor (Py-WFS), like the shearing and the curvature wavefront sensors, take advantage of the full telescope aperture, which has a diffraction limit much better that the subapertures of a Shack-Hartmann wavefront sensor. This induces a demonstrated gain by factors of 5 or higher, in the condition of operation envisaged for CaNaPy. It is to be noted that with poor seeing condition, a Shack-Hartmann wavefront sensor has to reduce the size of the subapertures projected on the pupil, enhancing the diffraction problem disadvantage. With CaNaPy it will be the first time that a Py-WFS is used in conjunction with a sodium LGS. Also in the downlink IR WFS, the use of a Pyramid WFS will be beneficial compared to a high order Shack-Hartmann, especially in tough seeing conditions when the Fried parameter $r_{0}$ is small (see Figure 2). In practice, as a rule of thumb the Py-WFS is advantageous over the SH-WFS whenever the reference target size is smaller than the diffraction of the SH-WFS subaperture.

The Py-WFS, like the curvature WFS, has non linearities which have been tackled in the control system on deployed systems from Arectri Observatory. Recently other groups have published adaptive modal compensation ${ }^{16}$ [ Deo 2019] and gain tracking ${ }^{17}$ [Chambouleyron 2021]. The implementation on CaNaPy of Py-WFS gain control methods is planned.

Numerical simulations comparing SH-WFS and Py-WFS operation on the LGS downlink performance of the CaNaPy system have been done using the public simulation code YAO developed by F. Rigaut, now at ANU. The code cannot yet simulate the uplink pre-compensation, although an upgrade is in progress. The results on Long Exposure Strehl ratio are shown in Table 1.

Table 1: Comparison of long exposureStrehl ratios obtained with CaNaPy LGS-AO. The better performance of the Py-WFS is evident, for all cases simulated: seeing of 0.7 " and 1 ", $1 \mathrm{kHz}$ and $2 \mathrm{kHz}$ operation.

- Tenerife Cn2 and wind median profiles

- Uplink not simulated yet in YAO (in progress)

- LGS Py-WFS, 4 pxl/ro

- OCAM2S 240x240 pxl, 0.4 e- rms r/o noise

\begin{tabular}{|c|c|c|c|}
\hline & \multicolumn{3}{|c|}{ LE Strehl } \\
\hline & PWFS & SHWFS & $\Delta$ \\
\hline $1 \mathrm{kHz} \quad 0.7^{\prime \prime}$ seeing & 0.692 & 0.535 & 0.157 \\
\hline $1 \mathrm{kHz} 1.0^{\prime \prime}$ seeing & 0.515 & 0.395 & 0.120 \\
\hline $2 \mathrm{kHz} 0.7^{\prime \prime}$ seeing & 0.795 & 0.602 & 0.193 \\
\hline $2 \mathrm{kHz} 1.0^{\prime \prime}$ seeing & 0.669 & 0.468 & 0.201 \\
\hline
\end{tabular}

A live demonstration of the simulation was given in the oral talk presentation at this conference.

ii https://www.highfinesse.com 
Another advantage of the Py-WFS is that it is not only sensing phase, but also scintillation. Which is an important parameter in the 24/7 OFL application. The image of the four pupils can be summed up to produce a map of the pupil intensity distribution, i.e. a map of the scintillation at AO loop frame rates, as can be seen in Figure 6 (see caption for details).
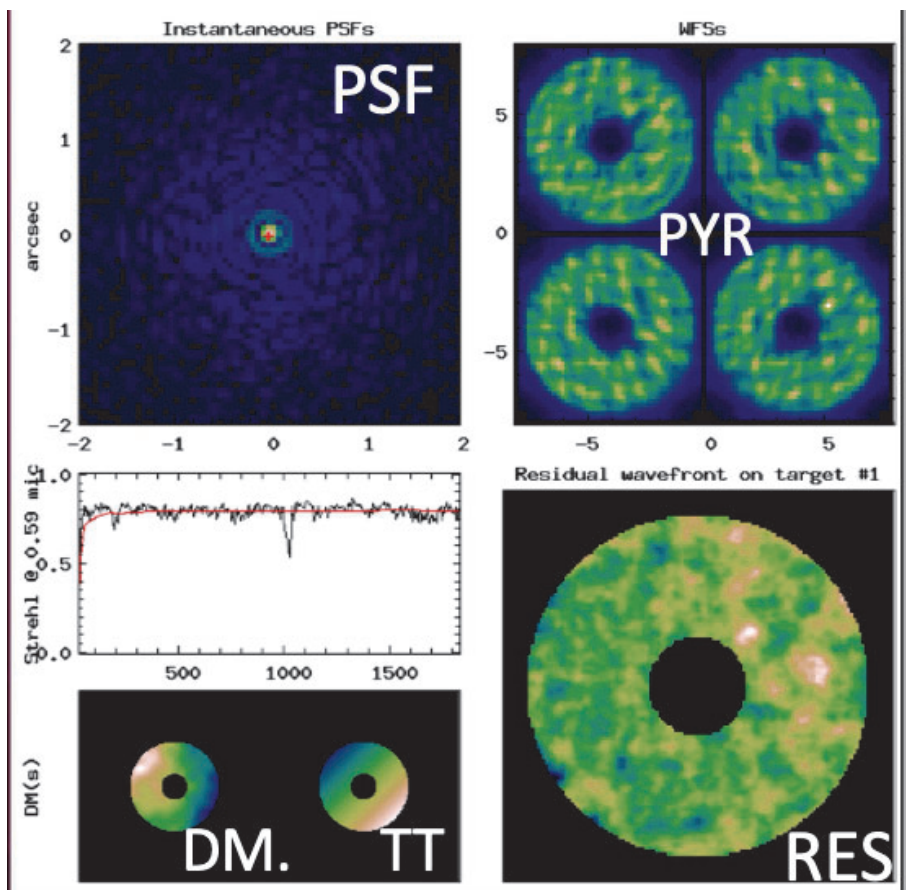

Figure 6: YAO LGS-AO numerical simulation. The display panel shows the instantaneous PSF (upper left), the DM and TT commands (lower left), the Image on the WFS camera of the four pupils from the Pyramid (upper right, OGS primary mirror), and the istantaneous residual wavefront error after correction (lower right). The sum of the four pupils gives at each loop frame the intensity map of the pupil, i.e. the scintillation can be observed and sensed with the Py-WFS.

\subsection{Laser Pointing Camera}

The laser pointing camera (Astrel Instruments) is a valuable tool when operating CaNaPy in LGS-AO. It will be used at OGS attached to the side telescope of the Optical Tube Assembly, as part of $\mathrm{CaNaPy}$. The camera has been developed by INAF with Astrel Instruments ${ }^{\text {iii }}$, for the ESO VLT 4LGSF ${ }^{18}$.

The camera allows to accurately point the LGS, giving feedback to the control system, as well as the LGS flux, FWHM, position offset.

A version capable of seeing also the IR uplink beam at $1064 \mathrm{~nm} \mathrm{~s}$ being studied for the OGS, to be able to automatically verify the superposition/offset of the 589nm and 1064nm uplink laser beams.

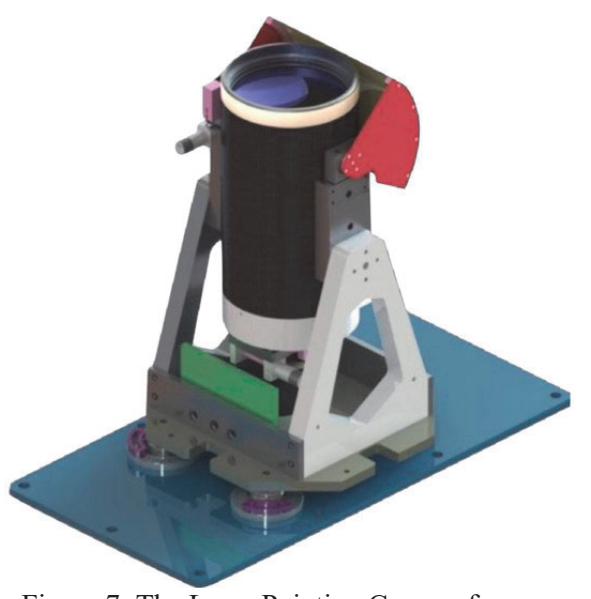

Figure 7: The Laser Pointing Camera from Astrel Instruments, as made for 4LGSF system of the VLT AOF.

iii https://www.astrel-instruments.com/astrel-pro 


\subsection{Axicons}

The double axicon system of CaNaPy is to transform a Gaussian laser beam into a ring, or donut, parallel beam. This allows to propagate the $\mathrm{CaNaPy}$ laser beam on a traditional reflective telescope with central obstruction, with minimal intensity losses, still retaining the full aperture advantages. In Figure 4 the intensity distribution after the double axicon is shown on the right side. A single axicon behavior is described by a Bessel-1 function ${ }^{19}$. Two double-axicons are necessary, one to work at $589 \mathrm{~nm}$ for the LGS uplink propagation without losses due to the ESA OGS central obstruction, and one to work at $1064 \mathrm{~nm}$, for the OFL full pupil propagation option. The fused silica axicons have antireflection coatings, with an extremely good surface roughness and glass homogeneity. They are used with the high-power laser beams, at 589 and $1064 \mathrm{~nm}$.

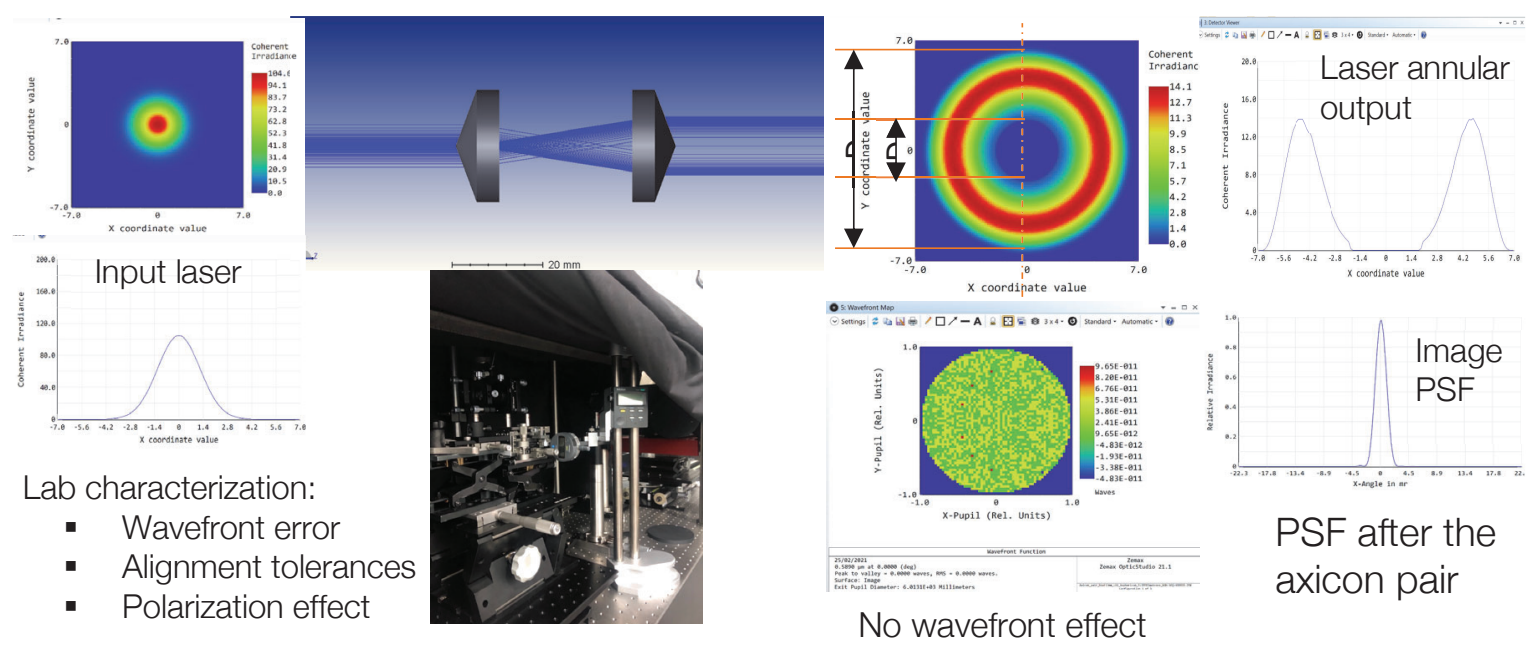

Figure 8: modeling the double axicon unit, using non-sequential surfaces in Zemax. The gaussian input beam (left plot) enters the double axicon cones and exit with an annulus. The CaNaPy axicons are made by Asphericon. In the optical lab the wavefront quality, the alignment tolerances and the Axicon effects on polarization have been tested and verified.

The double axicon system for CaNaPy is developed by Noelia Martinez, from the group of Prof. Celine d'Orgeville at $\mathrm{ANU}^{\mathrm{i}}$, in scientific collaboration with ESO. The mechanical system for the mount of the axicons has to take into account its sensitive alignment requirements.

\section{OFL UPLINK SIMULATION}

The uplink numerical simulation of the OFL uplink beam to be obtained with CaNaPy in closed loop is being done by Durham University members of the CaNaPy team, using physical optics propagation. Preliminary results are shown in Figure 9. The simulation shows the results of pre-compensation, for a 1550nm OFL laser illuminating a LEO satellite at $500 \mathrm{~km}$ above Earth, with a point ahead angle of 10 " and $\mathrm{r}_{\mathrm{o}}(500)$ of $0.1 \mathrm{~m}$. The LEO satellite is at 30 degrees elevation, and the footprint of the IR laser on the OGS pupil is $28 \mathrm{~cm}$. The images shown are for few msec integration.

In agreement with the analytical results shown in Figure 2, the use of LGS-AO at 10" point ahead angle and at this turbulence conditions with full uplink precompensation will be highly beneficial. More work is ongoing to validate the simulations for the different cases.

iv Australian National University, Mt. Stromlo Observatory (Canberra) 


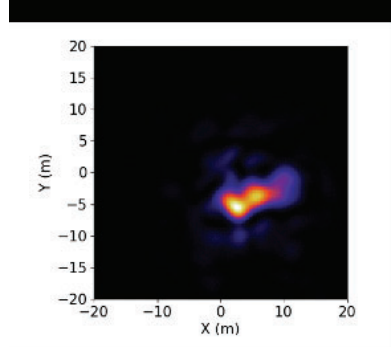

\section{Uncorrected}

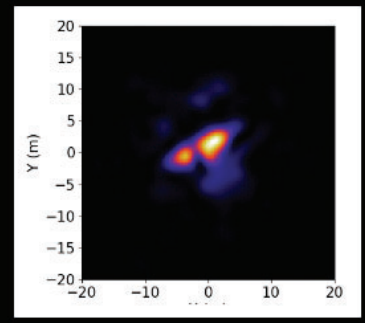

Tip/tilt only from satellite

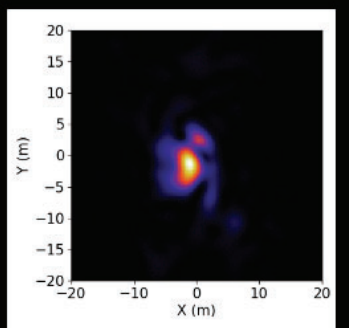

Full AO using satellite as reference

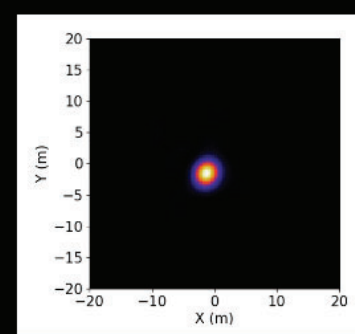

Full LGS AO, tip-tilt from satellite

Figure 9: Full physical optics simulation of the $1550 \mathrm{~nm}$ optical feeder link laser beam at a LEO satellite, pointing the OGS at $30 \mathrm{deg}$ elevation with ro $(1550)=0.1 \mathrm{~m}$ and median distribution of turbulence layers at Tenerife.

\section{CONCLUSIONS}

CaNaPy is an LGS-AO facility for the OGS telescope, built by ESO ${ }^{v}$, member state institutes and ESA. It is an LGS-AO system designed to operate in median seeing conditions. It will be installed at the Dome Coude', South Pier port of the OGS.

The CaNaPy 50W 589nm laser is based on a 100W RFA developed with MPBC (Canada), and a 589nm SHG unit developed by ESO with participation of HighFinesse $\mathrm{GmbH}$ and Toptica Projects GmbH. It shall be projected in monostatic configuration across the full OGS pupil, to form the laser guide star at the sodium mesospheric layer, $90 \mathrm{~km}$ a.s.l. Some details of the requirements for the laser have been given. The format requirements of the $589 \mathrm{~nm}$ laser were not trivial to reach. The evolution of the ESO $50 \mathrm{~W} 589 \mathrm{~nm}$ laser should be with the introduction of frequency chirping, to be decided after the preliminary tests are completed.

The scope of CaNaPy is to demonstate with field tests novel LGS-AO configurations useful for future astronomical adaptive optics at visible wavelengths, and for optical feeder links field tests in Satellite Optical Communications. It can operate $24 / 7$ as its Py-WFS is equipped with an extremely narrow band filter centered at the $\mathrm{D}_{2 \mathrm{a}}$ sodium line. However, it is to be noted that CaNaPy is dimensioned to cope with Fried parameters $r_{o} \geq 10 \mathrm{~cm}$, i.e. for median seeing conditions, and up to $2 \mathrm{kHz}$ loop operation. A demonstration of OFL is possible with CaNaPy as soon as it is installed and commissioned.

The goal of $100 \%$ sky coverage, by sensing the atmospheric tilt using the LGS, and operating in daytime with hard turbulence conditions, are part of the R\&D goals of the team, covering both astrophysics and Optical Communication needs.

The architecture of $\mathrm{CaNaPy}$ and its subsystems contains the experience cumulated by the participating institutes, over years of LGS-AO operation and system construction. Nonetheless there are new requirements and challenges which will have to be addressed, some of which have been presented

"ESO is bulding CaNaPy as part of the "Laser Guide Star Systems R\&D" work package, managed by one of the authors, within the ESO Technology Development Program activities, under the Directorate of Programmes. The agreement with ESA and the joint synergies effort for the OGS OFL field tests are managed for ESO by the Directorate of Engineering. 


\section{REFERENCES}

[1] Hippler, S., Kasper, M., Feldt, M., et al., "ALFA: three years of experience in adaptive optics with a laser guide star”, in Proc. SPIE Vol. 4007, p. 41-49 (2000).

[2] Wizinowich, P., Le Mignant, D., Bouchez, A. et al., “The W.M.Keck Observatory Laser Guide Star Adaptive Optics System: Overview”, PASP 118, 297-309 (2006).

[3] Bonaccini Calia, D., Friedenauer, A., Protopopov, V. et al.: "PM fiber lasers at 589nm: a 20W transportable laser system for LGS return flux studies", in Proc. SPIE 7736, Adaptive Optics Systems II, 77361 U (27 July 2010); doi: $\underline{10.1117 / 12.857991}$

[4] Feng, Y., Taylor, L., Bonaccini Calia, D. et al.: "39W narrow linewidth RamanFiber Amplifier with frequency doubling to 26.5W at 589nm", in Frontiers in Optics 2009/Laser Science XXV/Fall 2009 OSA Optics \& Photonics Technical Digest, (2009), paper PDPA4, https://doi.org/10.1364/FIO.2009.PDPA4

[5] Glenn, A., Tyler: "rapid evaluation of $d_{0}$ : the effective diameter of a laser-guide-star adaptive optics system", JOSA A, Vol. 11, Issue 1, pp. 325-338, (1994), https://doi.org/10.1364/JOSAA.11.000325

[6] P.-Y. Madec, R. Arsenault, H. Kuntschner, J. Kolb, J.-F. Pirard, J. Paufique, P. La Penna, W. Hackenberg, E. Vernet, M. Suárez Valles, N. Hubin: "Adaptive Optics Facility: from an amazing present to a brilliant future", SPIE Proc. Vol. 10703, Adaptive Optics Systems VI; 1070302 (2018) https://doi.org/10.1117/12.2312428

[7] Beckers, Jacques \& Cacciani, Alessandro. "Using Laser Beacons for Daytime Adaptive Optics", in Experimental Astronomy, 11. 133-143, (2001). https://doi.org/10.1023/A:1011140920850

[8] Beckers, Jacques. (2008). Laser guide stars for daytime thermal IR observations. Proc SPIE. 6986, (2008). https://doi.org/10.1117/12.801270

[9] Foy, R., Tallon, M., Friedman, H.W. et al.: "Polychromatic guide star", Proc. SPIE Vol. 3343, (1998). https://doi.org/10.1117/12.321566

[10] Esposito, S., Ragazzoni, R., Riccardi, A. et al.: “Absolute tilt from a Laser Guide Star: a first experiment”, in Experimental Astronomy V.10, Is. 1, p. 135-145 (2000)

[11] Roberto Ragazzoni: "Propagation Delay of a Laser Beacon as a Tool to Retrieve Absolute Tilt Measurements", ApJ Letters v.465, p.L73 (1996). doi: https://doi.org/10.1086/310139

[12] Bonaccini Calia, D., Guidolin, I., Friedenauer, A., et al.: "The ESO transportable LGS unit for measurements of the LGS photon return and other experiments", SPIE Proc. Vol. 8450, Modern Technologies in Space- and Ground-based Telescopes and Instrumentation II, (2012); https://doi.org/10.1117/12.926898

[13] Alaluf, D., Centrone, M., Bonaccini Calia, D., et al.: "Paving the way to Daytime Optical Feeder Links based on LGS assisted Adaptive Optics", this conf (2021)

[14] Bonaccini Calia, D., Feng, Y., Hackenberg, W. et al.: "Laser Development for Sodium Guide Stars at ESO”, The Messenger 139, p. 12-19 (2010).

[15] Roberto Ragazzoni" "Pupil plane wavefront sensing with an oscillating prism", Journal of Modern Optics, Vol. 43, 289-293 (1996). https://doi.org/10.1080/09500349608232742

[16]Deo, V., Gendron, E., Rousset, G. and F.Vidal: “A telescope-ready approach for modal compensation of pyramid wavefront sensor optical gain”, Astronomy and Astrophysics 629

[17] Chambouleyron, V., Fauvarque, O., Sauvage, J.-F. et al: "The focal-plane assisted pyramid wavefront sensor: enabling frame-by-frame optical gains tracking”, Astronomy and Astrophysics (submitted March 2021). https://arxiv.org/pdf/2103.02297.pdf

[18] Centrone, M., Bonaccini Calia, D., Pedichini, F., Cerruto, A., Ricciardi, A. and F. Ambrosino, "Laser pointing camera: a valuable tool for the LGS-AO operations", in Proc. SPIE, Vol. 9909, id. 99095L 7 pp. (2016).

[19] Marc Couture and Michel Piche: "Focusing properties of an axicon pair", in Canadian Journal of Physics, 71(12), p. 70-78 (1993). http://doi.org/10.1139/p93-011 